\title{
Espacialização pelo método da Krigagem nas variabilidades pluvial, evapotranspiração e evaporação no Estado do Pernambuco - Brasil
}

Spacialization by the Krigage method in pluvial variabilities, evapotranspiration and evaporation in the State of Pernambuco - Brazil

Spacialización por el método Krigage en variabilidades pluviales, evapotranspiración y evaporación en el Estado de Pernambuco - Brasil

Raimundo Mainar de Medeiros ORCID: https://orcid.org/0000-0003-3455-9876 Universidade Federal Rural de Pernambuco, Brasil E-mail: mainarmedeiros@gmail.com

Romildo Morant de Holanda

ORCID: https://orcid.org/0000-0001-7945-3616 Universidade Federal Rural de Pernambuco, Brasil E-mail: romildomorant@gmail.com

Luciano Marcelo Fallé Saboya

ORCID: https://orcid.org/0000-0002-7586-6867 Universidade Federal de Campina Grande, Brasil E-mail: 1saboya@hotmail.com

Fernando Cartaxo Rolim Neto ORCID: https://orcid.org/0000-0002-6411-2058 Universidade Federal Rural de Pernambuco, Brasil E-mail: fernandocartaxo@yahoo.com.br

Wagner Rodolfo de Araújo

ORCID: https://orcid.org/0000-0001-7203-0338

Universidade Estácio de Sá, Brasil

E-mail: wagneraraujops@gmail.com

Manoel Viera de França

ORCID: https://orcid.org/0000-0003-4973-9327

Universidade Federal Rural de Pernambuco, Brasil

E-mail: manoelvieira.ufrpe@gmail.com

\section{Resumo}

Objetiva-se especializar pelo método da krigagem os elementos precipitação, evapotranspiração e evaporação prováveis ao nível de $75 \%$ de probabilidade para 187 municípios gerando suas cartas e informações para os tomadores de decisões governamentais. Utilizaram-se dados de precipitação e temperatura média para gerar a evapotranspiração e evaporação mensal pelo método do balanço hídrico segundo Thornthwaite, com média de 40 anos, realizou análise no tocante à sua consistência, homogeneização e no preenchimento de falhas pelo método da média ponderada pelo o inverso da distância ao quadrado em cada série. As cartas foram geradas com a interpolação dos dados prováveis obtidos da precipitação, evapotranspiração e evaporação, com o programa Surfer 8.0. O método empregado para a interpolação dos dados foi a krigagem ordinária. A análise realizada neste estudo representa uma aproximação das potencialidades em termos de clima, recursos hídricos e das reais necessidades de água para as principais atividades de importância socioeconômica, visualizadas através do balanço hídrico. A probabilidade de $75 \%$ nos índices pluviais foi levada nas estações verão e outono na região litorânea, zona da Mata e Agreste. Nas estações primavera e inverno as contribuições pluviais foram decorrentes dos efeitos locais, orografia e dos sistemas de escala locais ocasionando chuvas fracas a moderada em curto intervalo de tempo. Na falta de elementos meteorológica necessária para determinar a evapotranspiração, a equação de Thornthwaite pode ser utilizada com razoável precisão para estimativa da evapotranspiração na área estudada.

Palavras chaves: Elementos climáticos; Balanço hídrico; Espacialização de dados.

\footnotetext{
Abstract

The objective is to specialize, using the kriging method, the elements of precipitation, evapotranspiration and evaporation, likely at the $75 \%$ probability level for 187 municipalities, generating their letters and information for government decision makers. Precipitation and average temperature data were used to generate evapotranspiration and monthly evaporation using the water balance method according to Thornthwaite, with an average of 40 years.
} 
inverse of the squared distance in each series. The charts were generated by interpolating the probable data obtained from precipitation, evapotranspiration and evaporation, using the Surfer 8.0 program. The method used for data interpolation was ordinary kriging. The analysis carried out in this study represents an approximation of the potential in terms of climate, water resources and the real water needs for the main activities of socioeconomic importance, visualized through the water balance. The $75 \%$ probability of rainfall was taken in the summer and autumn seasons in the coastal region, in the Mata and Agreste zones. In the spring and winter seasons, rainfall contributions were due to local effects, orography and local scale systems causing weak to moderate rainfall in a short period of time. In the absence of meteorological elements necessary to determine evapotranspiration, the Thornthwaite equation can be used with reasonable precision to estimate evapotranspiration in the studied area.

Keywords: Climatic elements; Water balance; Data spatialization.

\section{Resumen}

El objetivo es especializar, utilizando el método kriging, los elementos de precipitación, evapotranspiración y evaporación, probablemente al nivel de probabilidad del 75\% para 187 municipios, generando sus cartas e información para los tomadores de decisiones gubernamentales. Se utilizaron datos de precipitación y temperatura promedio para generar la evapotranspiración y la evaporación mensual utilizando el método de balance hídrico según Thornthwaite, con un promedio de 40 años, inverso de la distancia al cuadrado en cada serie. Los gráficos se generaron interpolando los datos probables obtenidos de precipitación, evapotranspiración y evaporación, utilizando el programa Surfer 8.0. El método utilizado para la interpolación de datos fue el kriging ordinario. El análisis realizado en este estudio representa una aproximación del potencial en términos de clima, recursos hídricos y las necesidades reales de agua para las principales actividades de importancia socioeconómica, visualizadas a través del balance hídrico. El 75\% de probabilidad de lluvia se tomó en las temporadas de verano y otoño en la región costera, en las zonas de Mata y Agreste. En las temporadas de primavera e invierno, las contribuciones de las lluvias se debieron a los efectos locales, la orografía y los sistemas de escala local que causaron lluvias débiles a moderadas en un corto período de tiempo. En ausencia de elementos meteorológicos necesarios para determinar la evapotranspiración, la ecuación de Thornthwaite puede usarse con una precisión razonable para estimar la evapotranspiración en el área estudiada.

Palabras clave: Elementos climáticos; Balance hídrico; Espacialización de datos.

\section{Introdução}

Assis et al. (2015) asseguraram que as mudanças do clima vêm apassivando cada vez mais a problemática da insuficiência hídrica, sobretudo em áreas áridas e semiáridas do planeta e em especial o semiárido do Nordeste do Brasil (SNEB), por apresenta tendência à aridização, acompanhada de da alteração nos padrões pluviométricos, ocasionando redução da frequência e intensidade nos índices pluviais, ainda conforme os autores a região SNEB é caracterizada com elevada potencialidade para evaporação d'água, em função da grande disponibilidade de energia solar, temperaturas elevadas e da baixa umidade do ar.

As atividades antrópicas sem controles associadas às distribuições e as variabilidades pluviais vem trazendo consequências negativas a socioeconômicas e a sobrevivência humana. A crescente intervenção humana ao meio físico vem aumentando substancialmente o grau de risco dos locais em relação a episódios que podem se tornar desastres (Nunes, 2016).

A análise de tendência em séries climáticas de precipitação vem sendo utilizadas visando estimar ocorrências de mudança climática locais, Silva et al. (2013). A tendência climática é uma mudança caracterizada por um aumento e/ou redução nos valores médios no período estudado (Back, 2011).

Os conhecimentos da importância da pluviometria são fundamentais na constituição do clima de uma determinada região, atuando, sobretudo na umidade do ar, balanço de água no solo e na temperatura. Sendo a variável meteorológica com maior ligação com o balaço hídrico, possibilitando determinar o excesso ou escassez hídrica de uma região (Silva et al., 2007).

De acordo com Vieira et al. (2010) a precipitação de determinado local pode ser estimada, dentre outras formas, em termos probabilísticos, mediante modelos teóricos de distribuição ajustados a uma série de dados. Silva et al. (2007), dentre os modelos de distribuição destaca-se o da distribuição Weibull que demonstra resultados satisfatórios, não apresentando diferença significativa quando comparado com outros métodos de distribuição para a estação chuvosa. 
A região Nordeste do Brasil (NEB) apresenta grandes variações espaciais e temporais de precipitação ao longo do ano (Nóbrega et al., 2014). Menezes et al, (2016) afirmaram que a grande variabilidade anual e interanual da precipitação, são características marcantes na região do NEB, provocando prejuízos sócios econômicos aos Estados.

Basicamente, a evapotranspiração $(E T P)$ representa a chuva teoricamente necessária para não faltar nem sobrar água no solo, e é utilizada na determinação do balanço hídrico para a identificação dos períodos de excesso ou escassez de água (CamargO et al., 1999; Barros et al., 2012; Costa et al., 2015).

Medeiros et al, (2016) em estudos sobre a ETP no município de Campina Grande - PB observaram que a ETP anual é de 1076,8 mm, com variações de 105,4 mm no mês de dezembro a 71,3 mm no mês de agosto, este valor no mês de agosto é menores devido a característica do final do inverno no Hemisfério Sul. O estudo também relata que a influência local tem grande contribuição sobre o aquecimento pontual, o chamado "aquecimento urbano" que se pode constatar nitidamente na área em estudo.

Pereira et al, (2012) relataram que a mesorregião do Sertão Paraibano, apresenta uma maior taxa de água evapotranspirada, quando comparada as outras regiões. Os mesmos descreveram que, as mesorregiões do Sertão, Borborema e Agreste, possuem um baixo índice de precipitação, quando comparada à alta evapotranspiração anual ocorrida, sendo o maior volume pluviométrico advindo da região Litoral do Estado.

Costa et al, (2015) computaram as oscilações da evaporação no tanque classe "A" no município de Teresina- PI e realizaram comparação com as mudanças na urbanização ocorrida no período estudo, encontrando alterações nos índices evaporativos em face da ocupação do Homem e suas respectivas modificações no espaço. O bloqueio do vento devido ao crescimento horizontal está contribuindo para a redução da evaporação (EVR), o contrário ocorre quando chove, não havendo escoamento superficial e ao término da precipitação os índices evaporativos ocorrem em maiores proporções devido à troca de calor. Verificaram-se ainda que as oscilações de menores e maiores valores ocorridos, com destaque para as décadas 19761985 e 1986-1995 que apresentaram as menores variações. A década de 2006-2011 no mês de outubro apresentou a maior flutuação dos períodos estudados. Os índices evaporativos tiveram maiores significâncias a partir da década de 1996, devido à verticalização urbana, alteração da área vegetal, compactação do solo com a pavimentação, aterramento de lagoas e eutrofização dos espelhos da água.

Estudos recentes têm demonstrado que as principais causas potenciais da redução da evaporação se evidenciam com as reduções generalizadas nos índices da temperatura do ar, da radiação solar, as mudanças associadas ao déficit de pressão de vapor e velocidade do vento (Roderick et al., 2007; Cong et al., 2009; Fu et al., 2009; McVicar et al., 2012).

Evapotranspiração terrestre é um dos componentes mais importantes do ciclo hidrológico, afetando o equilíbrio de água na superfície da Terra. É também uma das variáveis meteorológicas que é muito aplicada à tomada de decisão em hidrologia, agroecologia, irrigação e outras áreas afins (Fu et al., 2009; Roderick et al., 2009). Observações em todo o mundo com diferentes climas mostram que a demanda evaporativa medida utilizando-se tanques de evaporação tem reduzido ao longo dos últimos cinquenta anos. As quedas nos índices de evaporação para vários períodos desde o ano 1950 têm sido observadas em diversos países em ambos os hemisférios incluindo os EUA, a ex-União Soviética (Golubev et al., 2001), Itália (Moonen et al., 2002), Austrália (Roderick et al., 2004), Nova Zelândia (Roderick et al., 2005), Canadá (Burn et al., 2007), Índia (Jhaiharia et al., 2009) e China (Liu et al., 2010).

A metodologia da krigagem se distingue de diversos procedimentos de interpolação pelo formato de atribuição dos pesos. Yamamoto et al, (2015) diz que para compreender a oscilação espacial do processo aleatório subjacente, deve-se levar em consideração a possibilidade de que o valor em cada ponto no espaço está conexo com valores obtidos em pontos situados a certa distância, sendo razoável supor que a influência é tanto maior quanto menor for a distância entre os pontos. 
Kozmhinsky et al, (2018) marcaram as condições climáticas da insolação no Estado Pernambucano através da interpolação, elaborando mapas representativos da distribuição mensal e anual, além da variabilidade baixa e alta da insolação pelo método da krigagem.

Jakob (2012) afirmou que a metodologia da krigagem é aferida como um método de interpolação de dados tabular e a disposição geográfica para que se realizem os cálculos. Utilizando o princípio da Primeira Lei de Geografia de Tobler, que descreve que as unidades de análise próximas entre si são igualmente parecidas com as unidades distantes. A krigagem utilizase de funções matemáticas para juntar pesos maiores nas posições próximas aos pontos amostrais e pesos menores nas posições distantes, criando assim os novos pontos interpolados com base nessas combinações lineares de dados.

Objetiva-se especializar pelo método da krigagem os elementos climáticos precipitação, evapotranspiração e evaporação prováveis ao nível de 75\% de probabilidade para 187 municípios Pernambucano gerando suas respectivas cartas e gerando informações para os tomadores de decisões governamentais.

\section{Metodologia}

Um dos menores estados do Brasil em extensão territorial, Pernambuco possui grande diversidade de paisagens: planaltos, serras, brejos, semiaridez e belíssimas praias. O relevo é quase regular, sendo formado de planície litorânea cerca de $76 \%$ dele. À medida que deslocamos para o interior, encontram-se picos de montanhas ultrapassando os 1000 metros de altitude.

A cobertura vegetal é diversificada, com matas, manguezais, cerrados e caatinga. A vegetação litorânea predomina coqueiros, mangues e arbustos. A floresta tropical fica onde originalmente havia Mata Atlântica. Nas regiões do agreste e sertão pernambucano predomina a vegetação caatinga. Quanto à hidrografia, há muitos rios, principalmente na Região Metropolitana de Recife, que conta com 14 municípios. Os principais rios do estado são Capibaribe e Beberibe, Ipojuca, Una, Pajeú, Jaboatão e o rio São Francisco, este último extremamente importante no desenvolvimento do sertão, uma vez que possibilita a distribuição de água para as regiões atingidas pela seca.

Medeiros (2016) mostrou que os sistemas meteorológicos provocadores e/ou inibidores de chuvas para o estado do Pernambuco que contribuem com índices pluviométricos de moderado a fraca intensidade são os vestígios de Sistemas Frontais no setor sul do estado em menor frequência, às contribuições das Zonas de Convergência do Atlântico Sul, formações dos aglomerados convectivos e a contribuição da Alta da Bolívia. A Zona da Convergência Intertropical, perturbação associada à expansão para o hemisfério sul do equador térmico (zona de ascensão dos alísios por convecção térmica) provocam chuvas de moderada a forte intensidade em quase toda a área norte do Estado, seguidamente das contribuições das formações dos Vórtices Ciclônicos de Altos Níveis, os Distúrbios Ondulatórios de Leste e a Brisas Marítimas e Terrestres, sendo estes últimos originados no Oceano Atlântico; as Ondas de Leste são comuns no outono/inverno, auxiliadas pelos ventos alísios de sudeste, as ondas atingem a costa oriental do Nordeste, provocando chuvas fortes (Figura 1). 
Figura 1 - Ilustrações dos fatores meteorológicos atuantes no estado do Pernambuco.

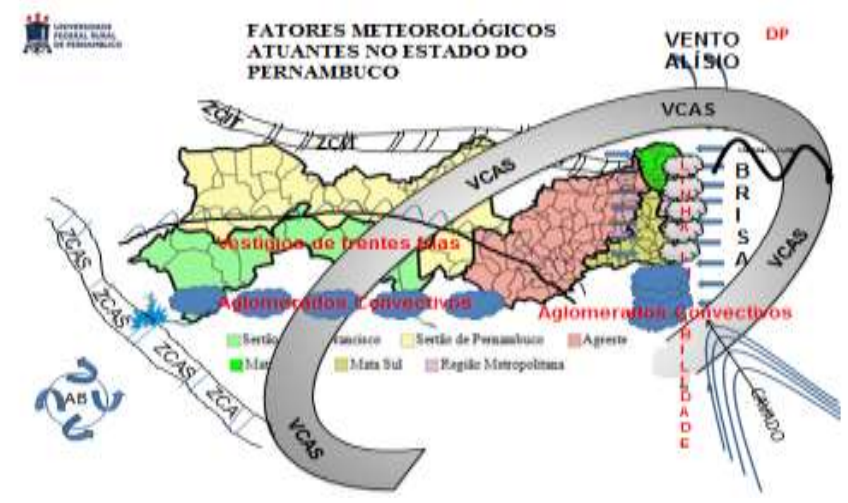

Fonte: Medeiros (2016).

Utilizaram-se dados de precipitação mensal e anual com média de 40 anos, fornecido pelo Instituto Nacional de Meteorologia (Inmet, 2019), Superintendência do desenvolvimento do Nordeste (Sudene, 1990) e da Agencia Pernambucana de água e clima (Apac, 2020) para 187 municípios que compõem o estado, para tanto se realizou análise no tocante à sua consistência, homogeneização e no preenchimento de falhas pelo método da média ponderada pelo o inverso da distância ao quadrado em cada série.

Utilizou-se dos valores das precipitações e das temperaturas média do ar para calcular o balanço hídrico climatológico dos 187 municípios gerando-se os dados de evapotranspiração e evaporação.

Calculou-se a precipitação, evapotranspiração e evaporação em nível de $75 \%$ de probabilidade referente às estações dos anos (primavera, outono, inverno e verão) de ser igualado ou superado aos seus valores históricos correspondentes à probabilidade dos elementos em estudo. Com os valores médios mensais prováveis calculados foram gerados suas cartas para todo o Estado Pernambucano. As cartas foram geradas com a interpolação dos dados prováveis obtidos da precipitação, evapotranspiração e evaporação, com o programa Surfer 8.0. O método empregado para a interpolação dos dados foi a krigagem ordinária.

Diferentes metodologias foram aplicadas para realização de interpolação para dados de evapotranspiração e precipitação tais como: média aritmética, interpolação natural, spline, ponderação da distância inversa, ponderação de distância angular, média ponderada pelo o inverso da distância ao quadrado e krigagem ordinária. Xavier et al. (2016).

A Figura 2 tem-se a variabilidade do clima de acordo com a classificação climática para o estado de Pernambuco com o modelo de Köppen. Destaca-se três tipos de climas encontrados neste estudo, "As" (clima tropical com estação seca de Verão) com maior predominância, o tipo "Bsh" (clima das estepes quentes de baixa latitude e altitude) com predomínio intermediário e o clima "Am" (Úmido. Clima de Bosque. Mês mais seco com a precipitação média inferior a 60 mm e a precipitação total anual superior a 10 vezes este valor) em menores proporções. 
Figura 2 - Classificação climática segundo o modelo de Köppen para o Estado do Pernambuco.

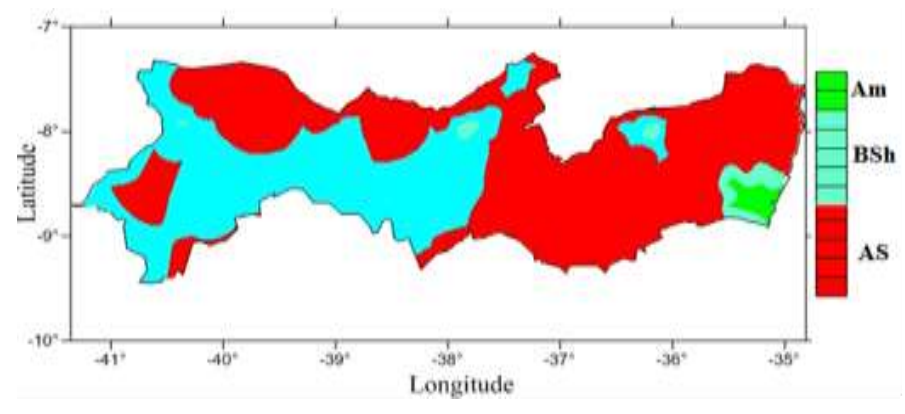

Fonte: Medeiros (2020).

Resultados análogos para estas classificações foram encontrados por Alvares et al. (2014) e Medeiros et al. (2018) na classificação climática para o Brasil em que destaca a costa do NEB, com clima "Am”, nos Tabuleiros Costeiros de João Pessoa, estendendo-se até a costa do Estado Pernambuco.

\section{Resultados e Discussão}

$\mathrm{Na}$ estação primavera (setembro-outubro-novembro) Figura 3 observam-se oscilações pluviais fluindo entre $2 \mathrm{~mm}$ a $52 \mathrm{~mm}$. Salienta-se que os maiores índices pluviais registram-se na zona litorânea e zona da Mata. Na região do agreste tem a faixa pluvial intermediaria. Nas regiões do sertão e alto sertão registra-as valores pluviométricos entre $2 \mathrm{~mm}$ a $17 \mathrm{~mm}$, nestas duas regiões estão iniciando-se as chuvas de pré-estação (chuvas que antecedem o período chuvoso). Destaca-se área isolada com índices pluviométricos prendidos no extremo oeste do estado, assim como na divisão da Paraíba e no agreste Pernambuco. Na região sul do estado os índices pluviais oscilam de 7 a 47mm. Estas flutuações concordam com os estudos de Marengo et al. (2012).

Figura 3 - Precipitação média provável a 75\% de probabilidade no Estado do Pernambuco na estação primavera.

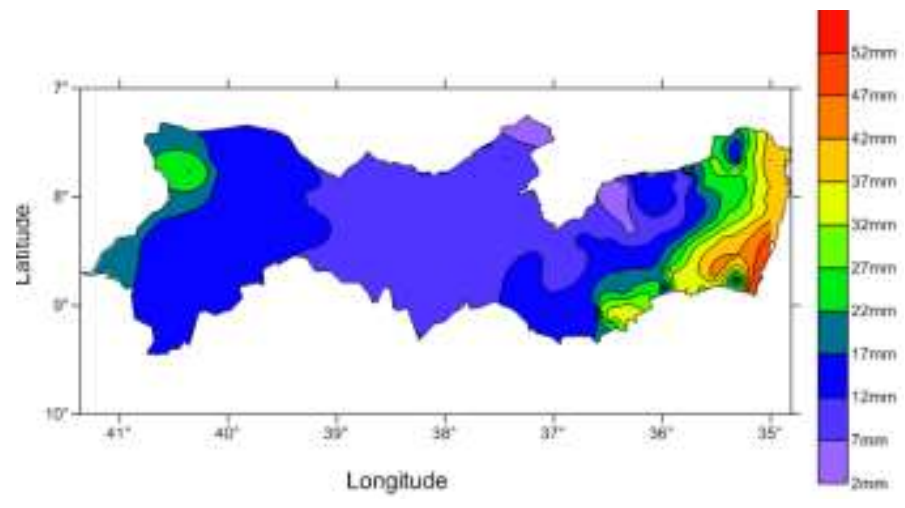

Fonte: Medeiros (2020).

Dayan et al. (2015) afirmaram que a intensidade dos eventos extremos de precipitação em determinada área decorre das características do meio, da localização e atuação dos sistemas meteorológicos de larga e média escala e das condições atmosféricas do local na escala espacial e temporal, que induzem a precipitação. Esses aspectos desempenham um papel importante na formação do resultado de tais eventos de chuva extrema. O que corroboram com o resultado do trabalho apresentado. 
A estação outono (março, abril e maio) (Figura 4) tem sua variabilidade pluvial média provável a $75 \%$ de probabilidade. Nas regiões: Litoral, Zona da Mata e Agreste registram-se os maiores volume pluviométricos oscilando entre 80 a 180mm, destaca-se núcleos isolados com índices pluviais na divisa da Paraíba e Alagoas e na região central e norte do agreste.

Nas regiões do sertão e alto sertão registram-se pluviometria oscilando entre $50 \mathrm{~mm}$ a $130 \mathrm{~mm}$, as áreas de menores índices pluviais pode ser observadas ao sul do estado na Figura 4, na posição norte-noroeste observa-se um núcleo pluvial com índices fluindo de $100 \mathrm{~mm}$ a 130.

Figura 4 - Precipitação média provável a 75\% de probabilidade no Estado do Pernambuco na estação outono.

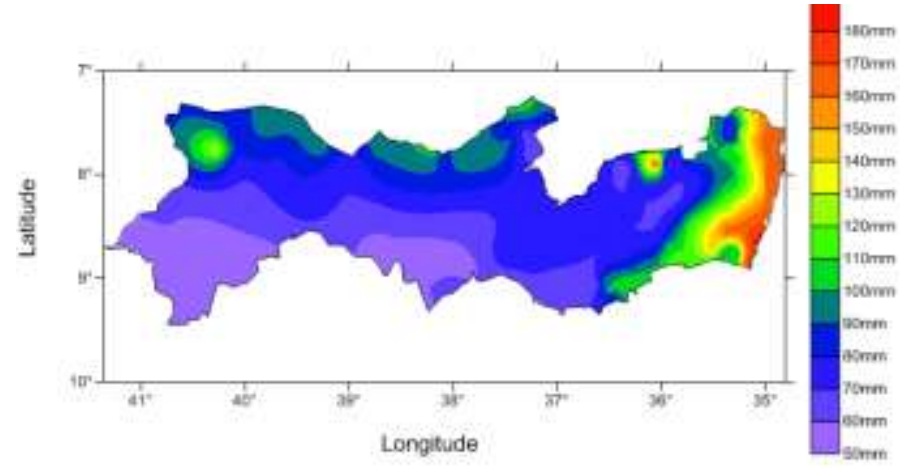

Fonte: Medeiros (2020).

Na Figura 5 tem-se a variabilidade da precipitação média provável a 75\% de probabilidade no Estado do Pernambuco na estação inverno (junho, julho e agosto). Nas regiões do alto sertão e sertão destacam-se núcleos pluviais isolados com oscilações entre $20 \mathrm{~mm}$ a $100 \mathrm{~mm}$, na sua grande maioria a precipitação observada é $20 \mathrm{~mm}$ no alto sertão e sertão. Nas regiões: Agreste, Zona da Mata e Litoral centra-se os altos valores pluviais. Resultados análogos que corroboram com o estudo apresentado foram obtidos por Nobrega et al, (2014).

Figura 5 - Precipitação média provável a 75\% de probabilidade no Estado do Pernambuco na estação inverno.

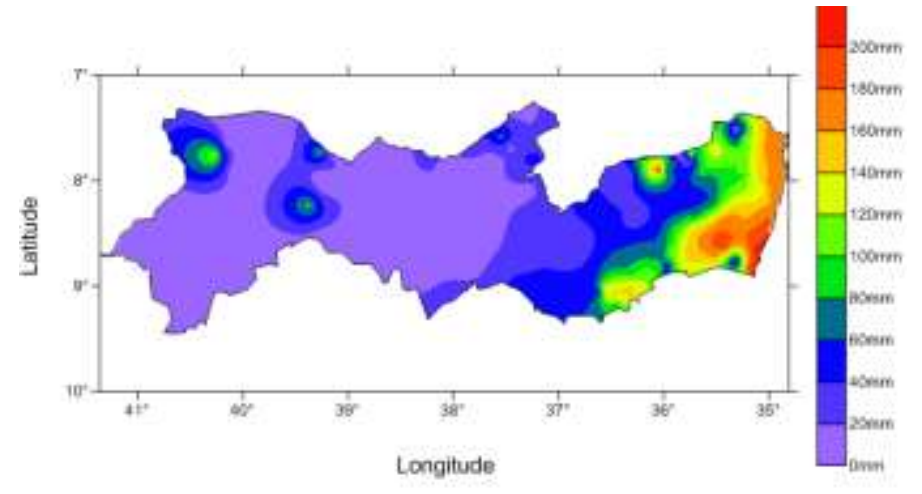

Fonte: Medeiros (2020).

A distribuição pluviométrica da estação verão (dezembro, janeiro e fevereiro) esta plotada na Figura 6 para o nível de probabilidade de $75 \%$. Nas regiões Alto Sertão e Sertão as oscilações pluviais fluem entre $28 \mathrm{~mm}$ a $82 \mathrm{~mm}$ destaca-se os núcleos pluviais isolados nas respectivas regiões. A região Agreste, Zona da Mata e Litoral tem suas oscilações pluviais fluindo entre $22 \mathrm{~mm}$ a $58 \mathrm{~mm}$. 
Estudos que vem a corroborar com os resultados deste trabalho podem ser revisto dos autores Nóbrega et al., (2014); Marengo et al. (2012).

Figura 6 - Precipitação média provável a 75\% de probabilidade no Estado do Pernambuco na estação verão.

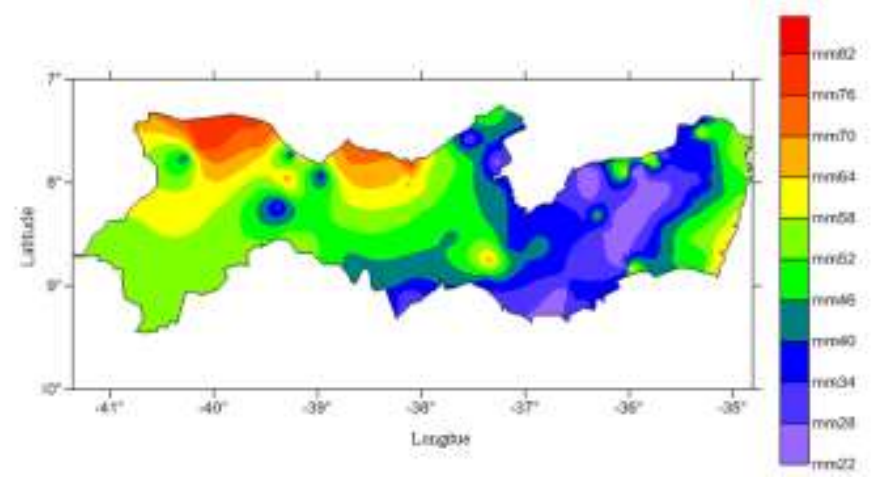

Fonte: Medeiros (2020).

Várias pesquisas como as dos autores (Marcuzzo et al., 2012; Eli et al., 2013; Ávila et al., 2014, Silveira et al., 2016), identificaram flutuações de variabilidade na precipitação devido as mudanças climáticas, estes estudos corroboram com os resultado discutidos.

As Figuras 7, 8, 9 e 10 representam as oscilações da evapotranspiração (ETP) para as estações primavera, outono, inverno e verão para o Estado do Pernambuco.

Nas Regiões: litorânea, Zona da Mata e Agreste a flutuabilidade da ETP fluem entre $61 \mathrm{~mm}$ a $91 \mathrm{~mm}$. Nas regiões: Sertão e Alto Sertão observam-se as oscilações da ETP fluindo entre $67 \mathrm{~mm}$ a $91 \mathrm{~mm}$, destaca-se o núcleo isolado da ETP na divisa da PB/PE com 67mm. (Figura 7). Resultado de interpolação análogos para o Estado do Piauí foram obtidos por Andrade Júnior et al. (2005).

Figura 7 - Evapotranspiração média provável a 75\% de probabilidade no Estado do Pernambuco na estação Primavera.

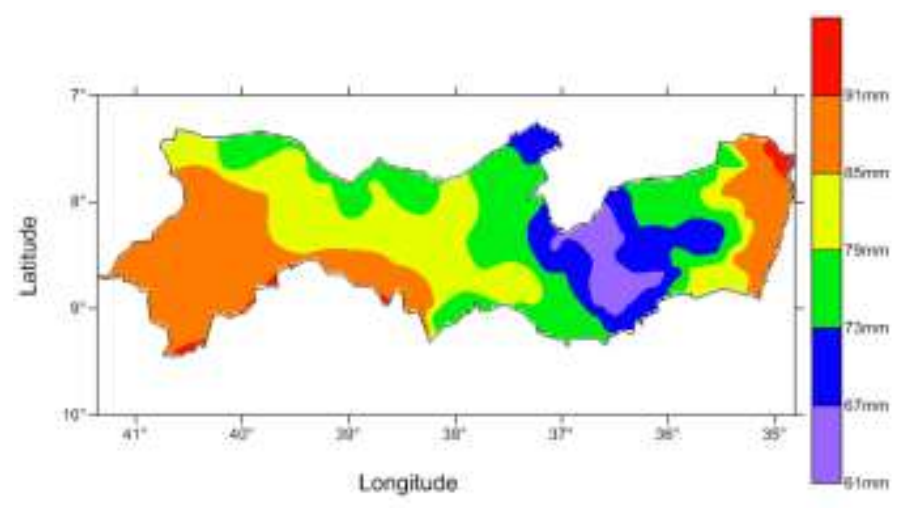

Fonte: Medeiros (2020).

Na Figura 8 têm-se as oscilações da Evapotranspiração média provável a 75\% de probabilidade no Estado do Pernambuco na estação Outono (março, abril e maio). Na região do Alto Sertão e Sertão os índices evapotranspirados oscilam entre $48 \mathrm{~mm}$ a $68 \mathrm{~mm}$. No agreste, Zona da Mata e Litoral a ETP na estação do outono fluem entre $48 \mathrm{~mm}$ a $68 \mathrm{~mm}$. Os fatores meteorológicos como cobertura de nuvem, intensidade vento, insolação, temperatura e umidade relativa contribuem para os registros destas variabilidades evaporativas. Tais flutuações estão em conformidade com o Estudo de Matos et al. (2014). 
Figura 8 - Evapotranspiração média provável a 75\% de probabilidade no Estado do Pernambuco na estação Outono.

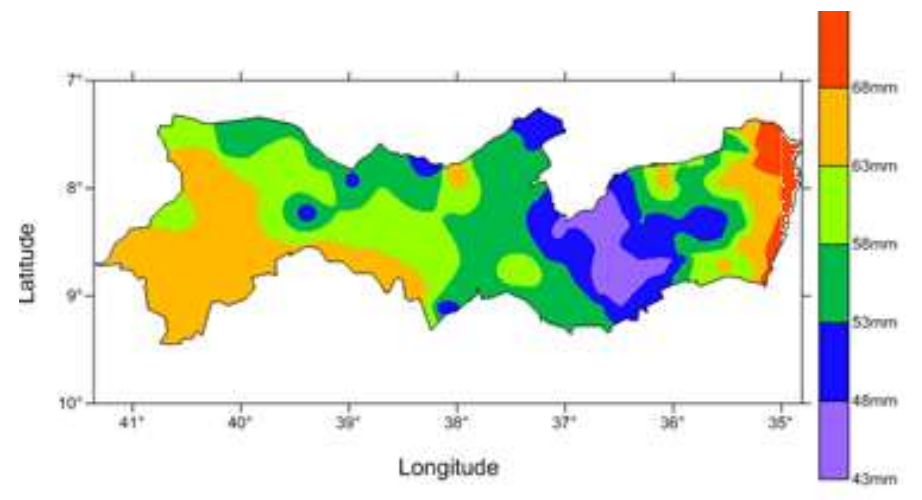

Fonte: Medeiros (2020).

A estação inverno (junho, julho, agosto) Figura 9 registram ETP oscilando entre 0mm a 72 mm, sendo final de período chuvoso nas regiões do litoral, zona da mata e agreste, nas regiões do Sertão e Alto Sertão estão no período seco. As chuvas ocorridas nas duas últimas áreas, são provocadas pelos elementos atmosféricos transientes auxiliados pelas trocas de calor aumentando ainda mais o poder evapotranspirado. Estes resultados estão de acordo com vários estudos realizados para o semiárido nordestino, conforme (Guedes Filho et al., 2011; Matos et al., 2014; Marengo et al. 2012).

Figura 9 - Evapotranspiração média provável a 75\% de probabilidade no Estado do Pernambuco na estação Inverno.

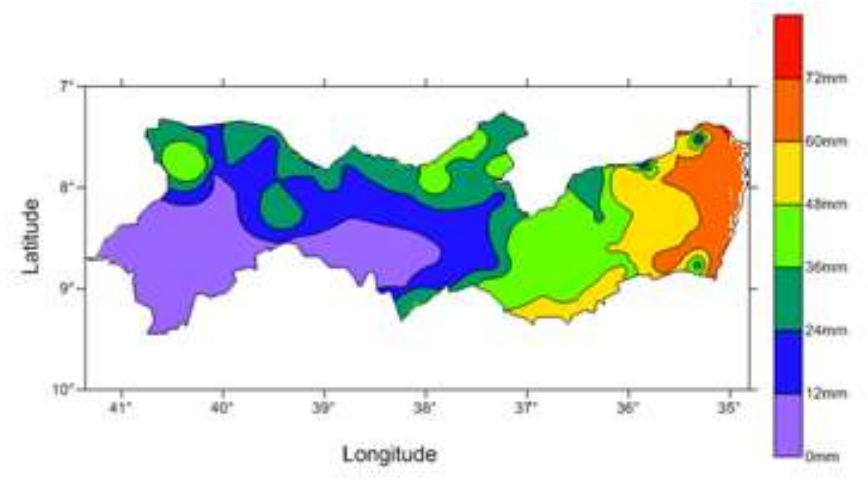

Fonte: Medeiros (2020).

A flutuação da ETP na estação Verão no estado do Pernambuco flui entre 70mm a 110mm. Na região do Alto Sertão e sertão a ETP oscila entre $78 \mathrm{~mm}$ a $110 \mathrm{~mm}$, destacam-se os núcleos isolados de máxima ETP nas áreas em analises, observamse ainda três núcleos de 70mm na divisa com a PB. As oscilações da ETP entre 102mm a 110mm na Zona litorânea, Na Zona da Mata registram flutuações de $92 \mathrm{~mm}$ a $102 \mathrm{~mm}$, na região do agreste fluem entre $70 \mathrm{~mm}$ a $94 \mathrm{~mm}$. Estas flutuações estão interligadas aos sistemas de larga, meso e da escala local e regional (Figura 10).

Nogueira et al. (2012) e Correia et al. (2011) concordam que a temperatura do ar se destaca entre as variáveis atmosféricas mais utilizadas no desenvolvimento de estudos de impactos ambientais com mudanças nos processos meteorológicos e hidrológicos que afetas os poderes evapotranspirativas e evaporativas da área estudada. 
Figura 10 - Evapotranspiração média provável a 75\% de probabilidade no Estado do Pernambuco na estação Verão.

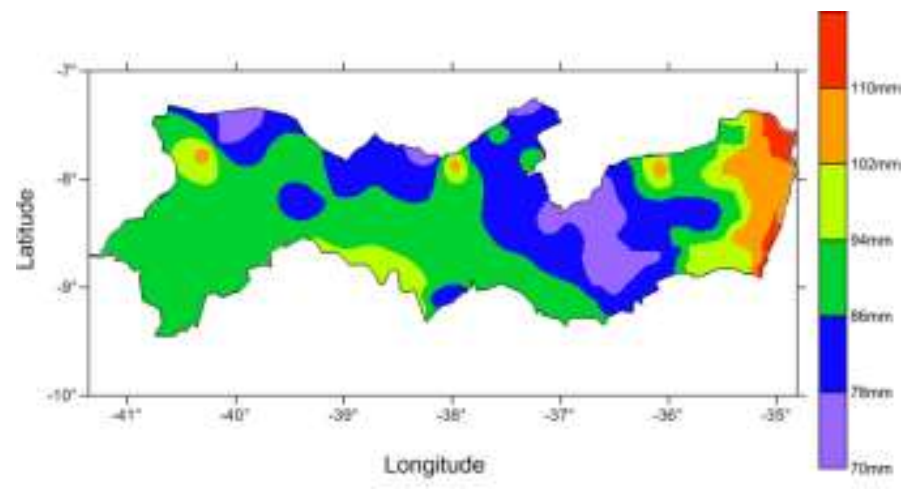

Fonte: Medeiros (2020).

Evaporação média provável a 75\% de probabilidade no Estado do Pernambuco na estação primavera está representada na Figura 11. Os maiores valores de EVR encontra-se nas regiões do Litoral e Zona da Mata, nas regiões do Agreste, Sertão e Alto Sertão registram os valores intermediários e os seus menores índico evaporativos. Destacam-se os núcleos insolados de EVR em todas suas regiões e de valores baixos, exceto na região do Agreste.

Figura 11 - Evaporação média provável a 75\% de probabilidade no Estado do Pernambuco na estação primavera.

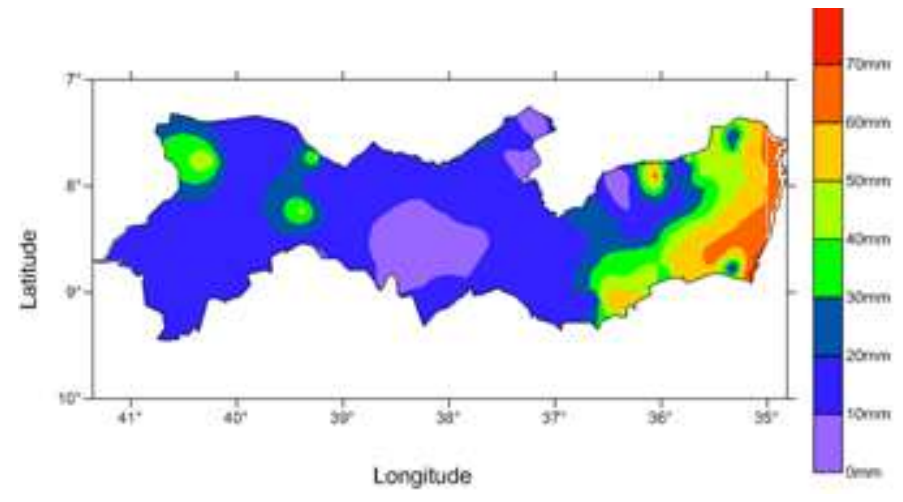

Fonte: Medeiros (2020).

Na estação outono (Figura 12) as variabilidades da EVR oscilam entre 50mm a 98mm. Nas regiões: Litorânea, Zona da Mata e agreste a EVR fluem de 58mm a 98mm com alguns núcleos em áreas isoladas. Com oscilações entre 50mm a 98mm e com núcleos isoladas de EVR de 98mm são as predominâncias da EVR nas regiões do Sertão e alto sertão.

Estudo como o de Barros et al. (2012) vem a corroborar com os resultados encontrados neste estudo.

Figura 12 - Evaporação média provável a 75\% de probabilidade no Estado do Pernambuco na estação outono.

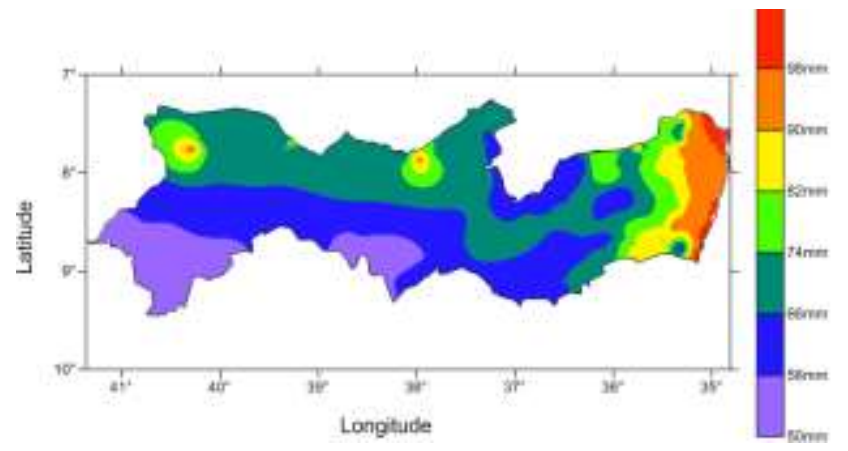

Fonte: Medeiros (2020). 
Na região do sertão e alto sertão registra-se os menores índices evaporativos com oscilações entre $0 \mathrm{~mm}$ a $48 \mathrm{~mm}$, nas regiões do Agreste, Zona da Mata e Litorânea registra-se os altos valores evaporativos da estação inverno (Figura 13).

Figura 13 - Evaporação média provável a 75\% de probabilidade no Estado do Pernambuco na estação inverno.

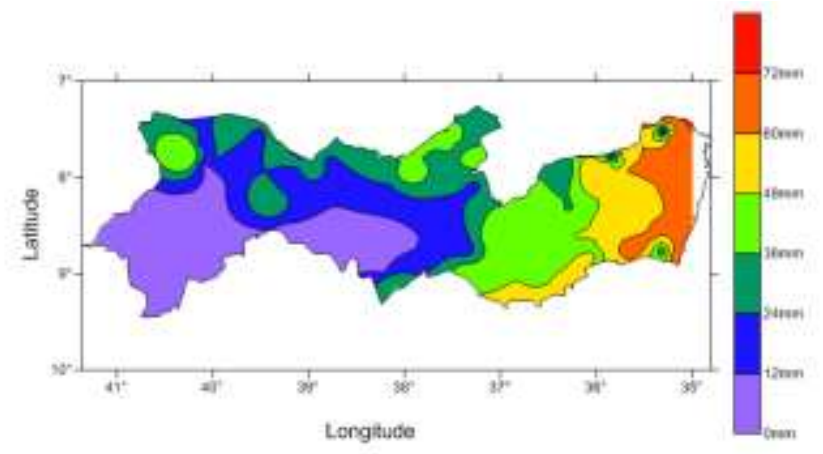

Fonte: Medeiros (2020).

Marengo (2012) afirmou que a região Nordeste do Brasil caracteriza-se pelo alto potencial evaporativo e evapotranspirativo em função da ampla disponibilidade de energia solar e das altas temperaturas. Aumentos de temperatura associados à mudança de clima decorrente do aquecimento global, sendo independente do que possa vir a acontecer com os índices pluviais, provocando maiores poderes evaporado dos lagos, córregos, lagoa, riachos açudes e reservatórios e máxima evaporação nas plantas. Isto é, a menos que haja aumento de chuvas, a água se tornará um bem mais escasso, com sérias consequências para a sustentabilidade do desenvolvimento regional.

Tem-se na Figura 14 a distribuição da evaporação (EVR) média provável a 75\% de probabilidade no Estado do Pernambuco na estação verão. Na estação verão a EVR oscila dentro do estado do Pernambuco entre $22 \mathrm{~mm}$ a $72 \mathrm{~mm}$. Nas regiões: Litoral, Zona da Mata e Agreste a EVR oscila entre 32mm a 72mm, destacam-se os núcleos isolados na divisa da PB e no extremo sul da região Litorânea. Na região Sertão e Alto Sertão a EVR flui entre 42mm e 72mm, salienta-se para estas áreas núcleos isolados.

Figura 14 - Evaporação média provável a 75\% de probabilidade no Estado do Pernambuco na estação verão.

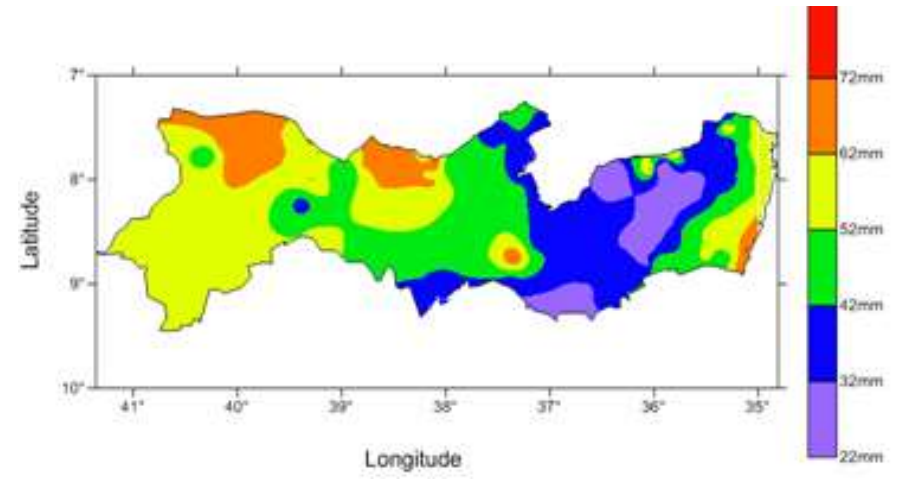

Fonte: Medeiros (2020).

Por outro lado, nos dias atuais é perceptível a importância das pesquisas que envolvem o estudo do clima na busca da construção de novos parâmetros de conhecimento e consequente aplicação nas diversas atividades humanas (agricultura, represamento de água, agropecuário, economia, comércio, lazer, irrigação, hidrologia entre outras ciências) que dependem dos dados e informações cada vez mais concisos sobre chuvas, secas temporais e eventos extremos, enfim, informações de médio e longo prazo geradas com um alto grau de acerto (Viana, 2010). 


\section{Conclusão}

A análise realizada neste estudo representa uma aproximação das potencialidades em termos de clima, recursos hídricos e das reais necessidades de água para as principais atividades de importância socioeconômica, visualizadas através do balanço hídrico.

A probabilidade de $75 \%$ nos índices pluviais foi levada nas estações verão e outono na região litorânea, zona da Mata e Agreste. Nas estações primavera e inverno as contribuições pluviais foram decorrentes dos efeitos locais, orografia e dos sistemas de escala locais ocasionando chuvas de fraca a moderada intensidade e em curto intervalo de tempo.

Na falta de elementos meteorológica necessária para determinar a evapotranspiração, a equação de Thornthwaite pode ser utilizada com razoável precisão para estimativa da evapotranspiração na área estudada.

A necessidade de uma interferência do poder público para a implementação de uma política de gestão da água, de forma que a população possa lucrar desse recurso de forma sustentável.

As avaliações da ETP/EVR mensais para o estado do Pernambuco mostrar conexão da localização geográfica e a topografia local.

A falta de arborização nos leitos das lagoas, lagos, rios, córrego, riachos, açudes e lençóis de águas, a edificação vertical e compactação de solo urbano e rural estão reduzindo o processo evaporativo e evapotranspirativo.

Evapotranspirou superior a chuva nas estações verão e primavera com 48,4\% e 27,3\%, ao passo que na EVR registrou-se cotas acima da chuva em 172,6\% na estação primavera. Os maiores valores de ETP/EVR ocorreram nas Regiões: Litoral, Zona da Mata e Agreste.

\section{Referências}

Alvares, C. A., Stape, J. L., Sentelhas, P. C., Gonçalves, J. L. M., \& Sparovek, G. (2014). Köppen's climate classification map for Brazil. Meteorologische Zeitschrift 22, 711-728.

Andrade Júnior, A. S., \& Medeiros R. M. (2005). Evapotranspiração de Referência mensal para o Estado do Piauí. Revista Brasileira de Engenharia Agrícola e Ambiental. 1, 560 - 564

APAC. Agencia Pernambucana de água e clima. (2019). www.apac.gov.br.

Assis, J. M. O., Souza, W. M., \& Sobral, M. C. M. Climate analysis of the rainfall in the lower-middle stretch of the São Francisco river basin based on the rain anomaly index. Revista Brasileira de Ciências Ambientais, 2,188- 202.

Ávila, P. L. R., Souza, E. B., Pinheiro, A. N., \& Figueira, W. S. 2014. Análise da precipitação sazonal simulada utilizando o regcm4 sobre o estado do Pará em anos de extremos climáticos. Revista Brasileira de Climatologia, 14, 50-75.

Back, A. J. (2011). Aplicação de análise estatística para identificação de tendências climáticas. Revista Pesquisa Agropecuária Brasileira, $36,717-726$.

Barros, A. H. C., Araújo Filho, J. C., Silva, A. B., \& Santiago. G. A. C. F. Climatologia do Estado de Alagoas. Boletim de Pesquisa e Desenvolvimento n.211. Recife: Embrapa Solos, 32p. 2012.

Burn, D. H., \& Hesch, N. W. (2007).Trends in evaporation for the Canadian Prairies. J. Hydrol. 336, 61-73.

Camargo, A. P. (1999).Balanço hídrico no Estado de São Paulo. IAC, 28p. (Boletim Técnico, 116).

Correia, M. F., Silva, F. S., Silva, A, M. R. S., \& Moura, M. S. B. (2011).Impacto da expansão agrícola na amplitude térmica diária em ambiente semiárido. Ciência e Natura, v. Suplementar, 311-314,

Costa, M. N. M., Medeiros, R. M., \& Gomes Filho, M. F. Variabilidade da evaporação no tanque classe "A" entre o período de 1976 a 2011 e suas comparações com as três décadas e meia e com o ano de 2011 no município de Teresina, Piauí. $7^{\circ}$ Workshop de Mudanças Climáticas do Estado de Pernambuco e $4^{\circ}$ Workshop Internacional sobre Mudanças Climáticas e Biodiversidade. Recife-PE, CD RON. 107-123. 2015.

Cong, Z. T., \& Yang, D. W., N. I, G. H. (2009).Does evaporation paradox exist in China? Hydrol. Earth Syst. Sci. 13, $357-366$

Dayan, U., Nissen, K. M., \& Ulbrich, U. (2015). Atmospheric conditions inducing extreme precipitation over the Eastern and Western Mediterranean. Natural Hazards and Earth System Sciences Discussions 3, 3687-3732.

Eli, K., Pitz, J. W., NeveS, L. O., Haveroth, R., \& Oliveira, E. C. (2013). Análise da distribuição da frequência de precipitação em diferentes intervalos de classes para Rio do Sul/SC. Enciclopédia Biosfera, 9, 106-113. 
Fu, G., Charles, S. P., \& Yu, J. A critical overview of pan evaporation trends over the last 50 years. Clim. Change 97, 193-214.

Golubev, V. S., Lawrimore, J. H., Groisman, P. Y., Speranskaya, N. A., Zhuravin, S. A., Menne, M. J., Peterson, T. C., \& Malone, R. W. Evaporation changes over the contiguous United States and the former USSR: A reassessment. Geophys. Res. Lett. 28, 2665-2668.

Guedes Filho, D. H., Santos Júnior, J. A., Costa Filho, J. F., \& Campos, V. B. Estimativa da evapotranspiração de referência para a cidade de Areia, Paraíba. Revista Brasileira de Agricultura Irrigada, 5(1), 37-47.

INMET. (2019). Normais climatológicas. Instituto Nacional de Meteorologia. Brasília - DF.

INMET - (2019). Instituo Nacional de Meteorologia. Banco de dados meteorológicos para ensino e pesquisa. <http://www.inmet.gov.br/portal/index.php?r=bdmep/b dmep>.

Jhaiharia, D., Shrivastava, S. K., Sarkar, D., \& Sarkar, S. Temporal characteristics of pan vaporation trends under the humid conditions of northeast India. Agr For. Meteorol. 149, 763-770, 2009.

Jakob, A. A. E. A krigagem como método de análise de dados demográficos. In: encontro da associação brasileira de estudos populacionais, 13. Ouro preto, Anais... Ouro preto, 2012.

KöppeN, W., \& Geiger, R. “Klimate der Erde. Gotha: Verlag Justus Perthes”. Wall-map 150cmx200cm. 1928.

Kozmhinsky, M., Medeiros, R. M., Holanda, R. M., \& Silva, V. P. Average insolation interpolated by the krigagem method for the state of Pernambuco Brazil. Journal of Hyperspectral Remote Sensing. 8(2). 334-344.

Liu, Q., Yang, Z., \& Xia, X. Trends for pan evaporation during 1959-2000 in China. Procedia Environmental Sciences, 2, 1934-1941.

Marengo, J. A., Nobre, C. A., Chou, S. C, Tomasella, J., Sampaio, G., Alves L. M., Obregon, G. O., Soares, W. R., Betts. R., \& Gillin, K. (2011).Riscos das Mudanças Climáticas no Brasil Análise conjunta Brasil-Reino Unidos sobre os impactos das mudanças climáticas e do desmatamento na Amazônia. 56p.

Marengo, J. A., Alves, L. M., Beserra, E. A., \& Lacerda, F. F. (2012).Variabilidade e mudanças climáticas no semiárido brasileiro. Recursos hídricos em regiões áridas e semiáridas. $303-422$.

Marcuzzo, F. F. N., Goularte, E. R. P., \& Melo, D. C. R. (2012).Mapeamento Espacial, Temporal e Sazonal das Chuvas no Bioma Amazônico do Estado do Tocantins. Anais. In: X Simpósio de Recursos Hídricos do Nordeste, 10.

Matos, R. M., Silva, J. A. S., \& Medeiros, R. M. (2014).Aptidão climática para a cultura do feijão caupi do município de Barbalha - CE. Revista Brasileira de Agricultura Irrigada, 8(6), 422-431.

Mcvicar, T., Roderick, M., Donohue, R., LI, T. L., Van NIel, T. G., Thomas, A., Grieser, J., JhajharaI, D., Himri, Y., Mohowald, N. M., Mescherskaya, A. V., Kruger, A. C., Rehman, S., \& Dinpashoh, Y. Global review and synthesis of trends in observed terrestrial near surface wind speeds: Implications for evaporation. J. Hydrol. 416-417, 182-205.

Medeiros, R. M. (1955).Planilhas do Balanço Hídrico Normal segundo Thornthwaite \& Mather

Medeiros, R. M. Fatores provocadores e/ou inibidores de chuva no Estado do Pernambuco.

Menezes, F. P., \& Fernandes, L. L. Análise de tendência e variabilidade da precipitação no Estado do Pará. Enciclopédia biosfera, Centro Científico Conhecer - Goiânia, 13(24), 1580-1591.

Medeiros, R. M., Holanda, R. M., Viana, M. A., \& Silva, V. P. (2018).CLIMATE Classification in Köppen model for the State of Pernambuco - Brazil. Revista de Geografia (Recife). 35, $219-234$.

Moonen, A. C., Ercoli, L., Mariotti, M., \& MasonI, A. (2002).Climate change in Italy indicated by agrometeorological indices over 122 years. Agr. For. Meteorol. 111, 13-27.

Nóbrega, J. N., Santos, C. A. C., Gomes, O. M., Bezerra, B. G., \& Brito, J. I. B. (2014). Eventos extremos de precipitação nas mesorregiões da Paraíba e suas relações com a TSM dos oceanos tropicais. Revista Brasileira de Meteorologia, 29(2), 197- 208.

Nogueira, V. F. B., Correia, M. F., \& Nogueira, V. S. (2012). Impacto do Plantio de Soja e do Oceano Pacífico Equatorial na Precipitação e Temperatura na Cidade de Chapadinha - MA. Revista Brasileira de Geografia Física, 5, 708 -724.

Nunes, L. H. (2016.). Compreensões e ações frente aos padrões espaciais e temporais de riscos e desastres. Territorium, (16), 179-189.

Pereira, M. C. A., Melo, D. F., Meló, R. G. C., Silva, R. F. B., \& Farias, S. A.R. Mesorregiões da Paraíba e suas necessidades de captação de água de chuva. In: Simpósio brasileiro de captação e manejo de água de chuva, 8. 2012. Campina Grande. Anais... Campina Grande: FIEP, 2012. p. 01-06.

Roderick, M. L., \& Farquhar, G. D. (2004). Changes in Australian pan evaporation from 1970 to 2002. Int. J. Climatol. 24, 1077-1090.

Roderick, M. L., \& Farquhar, G. D. (2005). Changes in New Zealand pan evaporation since the 1970s. Int. J. Climatol. 25, 2031-2039, doi:10.1002/joc.1262.

Roderick, M. L., Rotstayn, L. D., Farquhar, G. D., \& HobbinS, M. T. On the attribution of changing pan evaporation. Geophys. Res. Lett. 34, 10.1029/2007, GL031166.

Roderick, M. L., Hobbins, M. T., \& Farquhar, G. D. Pan evaporation trends and the terrestrial water balance. I. Principles and observations. Geogr. Compass 3/2, 746-760, 10.1111/j. 1749- 198. 2008. 00213.x, 
Research, Society and Development, v. 10, n. 2, e51010212758, 2021

(CC BY 4.0) | ISSN 2525-3409 | DOI: http://dx.doi.org/10.33448/rsd-v10i2.12758

Silva, R. B. (2013). Tendências de mudanças climáticas na precipitação pluviométrica no estado de Pernambuco. Dissertação (Mestrado) Pernambuco. Universidade Federal de Pernambuco.

Silva, J. C., Heldwein, A. B., Martins, F. B., Trentin, G., \& Grimm, E. L. Análise de distribuição de chuva para Santa Maria, RS. Revista Brasileira de Engenharia Agrícola e Ambiental, 11(1), 67-72.

Silveira, C. S., Souza filho, F. A., Martins, E. S. P. R., Oliveira, J. L., Costa, A. C., Nobrega, M. T., Souza, S. A., \& Silva, R. F. V. (2016). Mudanças climáticas na bacia do rio São Francisco: Uma análise para precipitação e temperatura. Revista Brasileira de Recursos Hídricos, 21, 416-428.

SUDENE. Superintendência do Desenvolvimento do Nordeste - Dados pluviométricos mensais do Nordeste - Série pluviometria 5. Estado da Paraíba. Recife, 239p. 1990

Viana, P. C. Estimativa e espacialização das temperaturas do ar mínimas, médias e máximas com base em um modelo digital de elevação para o Estado do Ceará. Trabalho de Conclusão de Curso - Curso de Graduação em Tecnologia em Irrigação e Drenagem, Instituto Federal de Educação e Ciência Tecnologia, Campus Iguatu - CE, 2010.

Vieira, J. P. G., Souza, M. J. H., Teixeira, J. M., \& Carvalho, F. P. Estudo da precipitação mensal durante a estação chuvosa em Diamantina, Minas Gerais. Revista Brasileira de Engenharia Agrícola e Ambiental,14(7).

Xavier, A. C., King, C. W., \& Scanlon, B. R. (2016). Daily gridded meteorological variables in Brazil (1980-2013). International Journal of Climatology, 36, 2644-2659.

Yamamoto, J. K., Landim, P. M. B. (2015). Conceitos e Aplicações.Oficina de textos, 